\title{
Effect of Adding Insulin Glargine on Glycemic Control in Critically III Patients Admitted to Intensive Care Units: A Prospective Randomized Controlled Study
}

This article was published in the following Dove Press journal:

Diabetes, Metabolic Syndrome and Obesity: Targets and Therapy

\author{
Nader D Nader (D) \\ Hadi Hamishehkar ${ }^{2}$ \\ Abdolreza Naghizadeh ${ }^{3}$ \\ Kamran Shadvar ${ }^{3}$ \\ Afshin Iranpour ${ }^{4}$ \\ Sarvin Sanaie ${ }^{5}$ \\ Francis Chang' \\ Ata Mahmoodpoor ${ }^{3}$
}

'State University of New York at Buffalo, Jacobs School of Medicine and Biomedical Sciences, Buffalo, NY, USA; ${ }^{2}$ Department of Clinical Pharmacy, Faculty of Pharmacy, Tabriz University of Medical Sciences, Tabriz, Iran; ${ }^{3}$ Department of

Anesthesiology and Critical Care, Faculty of Medicine, Tabriz University of Medical Sciences, Tabriz, Iran; ${ }^{4}$ Al Garhoud Private Hospital, Dubai, United Arab Emirates; ${ }^{5}$ Neurosiences Research Center, Aging Research Institute, Tabriz University of Medical Sciences, Tabriz, Iran
Correspondence: Ata Mahmoodpoor Anesthesiology, Tabriz University of Medical Sciences, School of Medicine, Tabriz, Iran

Email amahmoodpoor@yahoo.com

\begin{abstract}
Objective: We aimed to examine the effects of adding a longer-acting insulin glargine to existing glucose control on reducing blood-glucose fluctuations in an intensive care unit (ICU). Methods: A total of 110 patients randomly received adjuvant insulin glargine 15 IU/day (glargine) or placebo (control), in addition to daily infusion of insulin to maintain glucose levels at a target of 140-180 mg/dL. End points were mean and variance of blood glucose and frequency of hypoglycemia, hyperglycemia, ICU stay, and mortality. Data were analyzed with repeated-measures ANOVA and Mann-Whitney $U$ test.

Results: Average daily glucose level was significantly less in the glargine group than controls $(P<0.0001)$, while there was no difference in daily variance in blood glucose between the two groups. The duration of glucose concentrations being within the target range was identical between the glargine and control groups ( $16.6 \pm 4.9$ vs $16.4 \pm 4.6$ hours/ day, $P=0.844$ ) during the 7 days of admission. The frequency of hypoglycemia was greater in the glargine group and total duration of hyperglycemia ( $>180 \mathrm{mg} / \mathrm{dL})$ much longer among controls $(P<0.001)$. Similar mortality rates were observed in both groups, while ICU length of stay was 2 days shorter in the glargine group.

Conclusion: Addition of insulin glargine to routine protocols more effectively reduces glucose levels and decreases incidence of hyperglycemic episodes and regular insulin usage. This adjustment may be associated with decreases in duration of ICU stay or increases in hypoglycemic events.
\end{abstract}

Keywords: critically ill patients, dysglycemia, hyperglycemia, insulin glargine

\section{Introduction}

After the landmark study of van den Berghe et al in 2001 about the significant mortality benefit of tight glycemic control, many centers have tried to evaluate the feasibility of treating dysglycemia and maintaining normoglycemia in critically ill patients. ${ }^{1}$ Previous studies have demonstrated that hyperglycemia, hypoglycemia, and glucose variability are independent risk factors for mortality of dysglycemic patients in intensive care settings. ${ }^{2-4}$ Strict glycemic control is a time-consuming procedure and increases nursing workload and costs. ${ }^{5}$ Although there is controversy on setting a target for blood glucose (BG) levels, intravenous infusion of regular short-acting insulin is the preferred approach for controlling serum glucose in intensive care units (ICUs). ${ }^{6}$ On the other hand, long-acting insulin formulations are 
not generally considered because of the altered pharmacokinetic and pharmacodynamic properties of the drug. ${ }^{7}$ Insulin glargine has a lower peak effect, with an onset of action of around 2 hours. Although the risk of hypoglycemia is lower than other long-acting insulin preparation, this risk is not zero. ${ }^{8}$

Administration of insulin glargine with intravenous insulin for critically ill hyperglycemic patients may prove beneficial, based on the pharmacokinetics and pharmacodynamics of insulin glargine, which provide a stable plasma level of insulin with a long half-life when compared to other forms of insulin. ${ }^{9}$ This approach has been shown to be safe in pediatric patients with diabetic ketoacidosis. ${ }^{10,11}$ In 2012, a modelbased trial was designed to safely investigate the effect of glargine in critically ill patients before developing a final protocol. ${ }^{12}$ Thereafter, many trials of using insulin glargine for accurate management of dysglycemia in critically ill patients have been performed. Datta et al showed that hyperglycemia management was significantly better with the use of insulin glargine compared to regular insulin after a bariatric surgical procedure. ${ }^{13}$ They also reported that hypoglycemia is very infrequent. As there have been conflicting results about the routine practice for accurate management of hyperglycemia, in this study we evaluated adding a small dose of insulin glargine on the routine protocol in the short-term management of dysglycemia in critically ill patients admitted to ICUs. We hypothesized that adding glargine insulin to the existing insulin protocol would reduce daily variation in BG, reduce the mortality rate, and shorten the duration of stay in the ICU.

\section{Methods}

This was a randomized controlled trial (RCT), which was reviewed and approved by the Tabriz University of Medical Sciences Ethics Committee and conducted in accordance with the guidelines proposed in the Declaration of Helsinki. After written informed consent had been obtained from patients or their next of kin, 110 critically ill patients were enrolled. The study is registered under IRCT201608182582N14.

\section{Inclusion and Exclusion Criteria}

All patients who had been admitted to two universityaffiliated ICUs and candidates for insulin therapy for dysglycemia management were enrolled in this study. Exclusion criteria were allergy to insulin, intolerance of enteral feeding, pregnancy, any hypersensitivity to

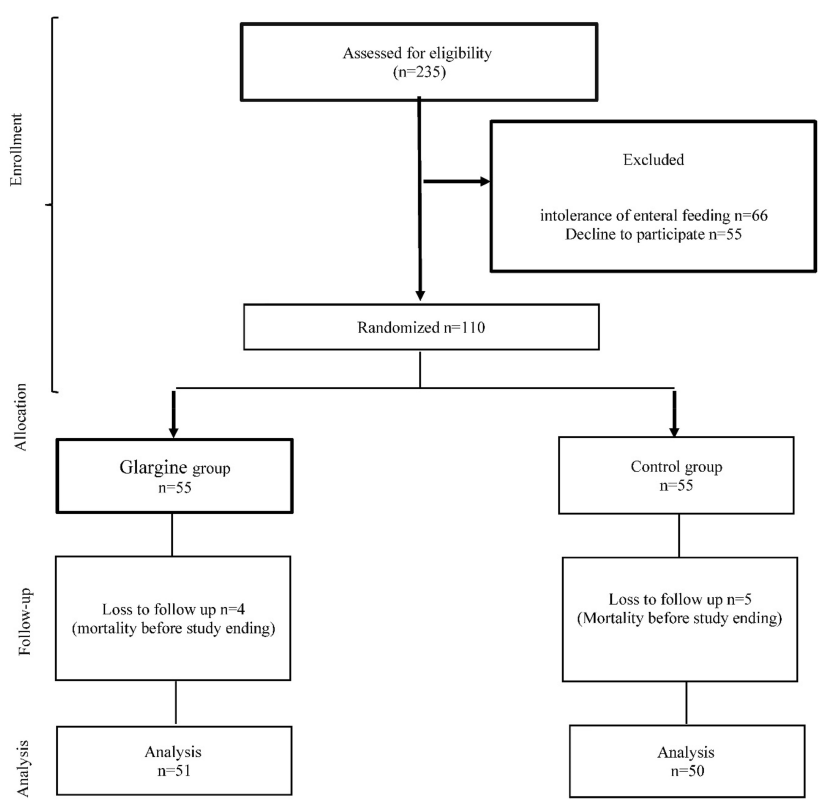

Figure I CONSORT flow diagram.

glargine insulin, patients $<18$ years of age, declining, to participate, and intolerance of enteral feeding (Figure 1).

\section{Interventions}

Patients were randomly allocated into two groups using simple randomization during their first 24 hours of ICU admission in this single-blinded RCT. Patients in the control group received continuous infusion of regular insulin (Actrapid HM; Novo Nordisk, Denmark) with a target BG level of 140-180 mg/dL. Insulin was infused continuously using a Perfusor FM (MFC) (B Braun, Darul-Ehsan, Malaysia) pump based on the formula BS - 140/20. Patients in the intervention group received continuous infusion of regular insulin plus 15 IU insulin glargine subcutaneously. The study duration was 7 days. Insulin was started when the BG level exceeded $180 \mathrm{mg} / \mathrm{dL}$.

\section{Definitions and Outcome Variables}

The BG target was set at $140-180 \mathrm{mg} / \mathrm{dL}$. In septic patients, because of the high prevalence of hypoglycemia, the lower limit of acceptable BG was $150 \mathrm{mg} / \mathrm{dL}$. Hyperglycemia was defined as BG $>180 \quad \mathrm{mg} / \mathrm{dL}$. Hypoglycemia was defined as $\mathrm{BG}<60 \mathrm{mg} / \mathrm{dL}$ and severe hypoglycemia as $B G<40 \mathrm{mg} / \mathrm{dL}$. Daily variance in $\mathrm{BG}$ was the primary outcome variable of this study and used for sample-size determination. Mean daily BG concentrations, duration of time in target, amount of regular insulin used for hyperglycemic crisis, duration with BG $>180 \mathrm{mg} /$ 
$\mathrm{dL}$, frequency of hypoglycemic events ( $\mathrm{BG}<60 \mathrm{mg} / \mathrm{dL}$ ) and severe hypoglycemia (BG $<40 \mathrm{mg} / \mathrm{dL}$ ), duration of ICU stay, and ICU mortality were the secondary outcome variables of the study.

\section{Blood Sampling and Glucose Measurement}

Blood samples were obtained from an indwelling arterial line placed in the upper extremity and analyzed by an automated chemical analyzer (Abbott, Chicago, IL, USA). Sampling was done every hour, and if four consecutive samples were in the normal range every 2 hours. If three consecutive samples were in the target range, the sampling interval was increased to 4 hours. We did not increase the sampling period to $>4$ hours because of hypoglycemic complications. Also, we measured BG every 12 hours in the laboratory to check the accuracy of our glucometer (cross-validation), and if the difference was $>15 \%$ we changed the device. If BG was $<70 \mathrm{mg} / \mathrm{dL}$, an insulin infusion dextrose $50 \%$ in water administered based on the formula $0.4 \times(100-$ BG $)$ in milliliters. During hypoglycemia, we used point-of-care glucometers (Accu-Chek Performa) to measure BG every 30 minutes until correction of hypoglycemia. The compliance of ICU nurses with the protocol was high (90\%). Mean BG level, $\mathrm{HbA}_{1 \mathrm{c}}$, mean regular insulin, and number of hypoglycemic events were noted for all patients.

\section{Nutrition and Routine Patient Care}

All patients were nourished via an enteral route with a target of $25 \mathrm{kcal} / \mathrm{kg}$ with standard formula (Ensure 1 $\mathrm{kcal} / \mathrm{mL}$; Abbott). All enrolled patients received $0.8 \mathrm{~g} / \mathrm{kg}$ protein daily throughout the study period. Total intake of calories and gastric residual volume were noted for all patients. They were considered intolerant to enteral nutrition if gastric residual volume was more than half the enteral formula administered during the prior 6 hours. If patients tolerated as high as $50 \%$ of their required calories through the enteral route, supplemental parenteral nutrition was added to meet their daily energy requirement. Patients were excluded from the study if they were unable to tolerate at least half their energy requirement through an enteral route. Routine respiratory care was provided for all patients. For those patients who were on mechanical ventilation, intravenous sedation was provided to help them tolerate placement of the endotracheal tube and for positive-pressure breathing. The Richmond Agitation Sedation Scale (RASS) was used to assess the level of sedation as per routine of inpatients at our ICUs.
Sedation was provided with intravenous infusion of midazolam titrated to maintain target RASS scores between -2 and 0 .

\section{Statistical Analysis}

Sample size was calculated based on Datta et $\mathrm{al}^{13}$ using the online sample-size calculator provided by the University of British Columbia (www.stat.ubc.ca). As mean BG level of patients after abdominal surgery with glargine insulin was $134 \pm 33 \mathrm{mg} / \mathrm{dL}$ and with regular insulin $154 \pm 33 \mathrm{mg} / \mathrm{dL}$ (SD $30 \mathrm{mg} / \mathrm{dL}$ ), sample size was calculated as 42 subjects per group, assuming $\alpha=0.05$ and power of 0.8 . Considering the loss of patients during the study, we included 50 patients per group. All data were analyzed with IBM's SPSS 24.0 . Patient characteristics at baseline are described as counts $(\%)$ for categorical variables. Categorical variables were examined with $\chi^{2}$ and Fisher's exact tests. For numeric variables, the Kolmogorov-Smirnov goodness-of-fit test was used for assessment of normal distribution of variables. Data with normal distribution were compared using independent $t$-tests and are presented as means $\pm \mathrm{SD}$, while those without normal distribution were analyzed using a nonparametric test (Mann-Whitney $U$ ) and aree presented as medians and IQR. Repeated-measures ANOVA was used for comparison of average daily $\mathrm{BG}$ and its variance with daily use of regular insulin. $P<0.05$ was considered significant.

\section{Results}

A total of 110 patients who had been admitted to two university-affiliated ICUs and undergone glycemic control with insulin therapy were enrolled in this study. Nine patients - four from the study group and five from the control group - were excluded for protocol deviation. Demographic characteristics of the patients are shown in Table 1. Our results showed that the two study groups were similar regarding age, sex, weight, body-mass index, diabetes mellitus, and comorbidities. $\mathrm{HbA}_{1 \mathrm{c}}$ showed no significant difference between the groups, nor did baseline lipid profile, total cholesterol, high- and low-density lipoproteins, and triglyceride concentrations. Patients in the two groups did not show any significant difference regarding baseline albumin, prescribed energy, or protein during the study protocol.

The average time that $\mathrm{BG}$ levels were within target $(140-180 \mathrm{mg} / \mathrm{dL})$ was $16.6 \pm 4.9$ hours/day in the glargine group and $16.4 \pm 4.6$ hours among controls $(P=0.844)$. 
Table I Patient Characteristics and Comorbidities in Treatment Groups

\begin{tabular}{|c|c|c|c|c|c|}
\hline \multirow[b]{2}{*}{ Sex, male/female } & \multirow{2}{*}{$\begin{array}{l}P \text {-value } \\
0.778\end{array}$} & \multicolumn{2}{|c|}{ Controls $(n=55)$} & \multicolumn{2}{|c|}{ Glargine $(n=55)$} \\
\hline & & $31 / 19$ & & $33 / 18$ & \\
\hline Age (years) mean $\pm S D$ & 0.273 & 63.2 & \pm 13.2 & 60.2 & \pm 14.9 \\
\hline Body weight (kg) & 0.110 & 65.8 & \pm 14.0 & 69.5 & \pm 8.3 \\
\hline Body-mass index $\left(\mathrm{gg} / \mathrm{m}^{2}\right)$ & 0.158 & 25.8 & \pm 4.5 & 27.0 & \pm 3.7 \\
\hline \multicolumn{6}{|l|}{ Admission diagnosis } \\
\hline Cerebrovascular accidents & 0.194 & 16 & $32 \%$ & 6 & $12 \%$ \\
\hline Neoplastic disease & & 9 & $18 \%$ & 13 & $26 \%$ \\
\hline Multitrauma & & 9 & $18 \%$ & 7 & $14 \%$ \\
\hline Respiratory failure & & 7 & $14 \%$ & 7 & $14 \%$ \\
\hline Spinal cord injury/surgery & & 3 & $6 \%$ & 5 & $10 \%$ \\
\hline Pulmonary thromboembolic events & & 3 & $6 \%$ & 3 & $6 \%$ \\
\hline Acute coronary syndrome & & 2 & $4 \%$ & 2 & $4 \%$ \\
\hline Sepsis & & 0 & 0 & 4 & $8 \%$ \\
\hline Major urological procedure/surgery & & I & $2 \%$ & 2 & $4 \%$ \\
\hline Poisoning & & 0 & 0 & 2 & $4 \%$ \\
\hline Enteral/parenteral feeding (nutrition) & 0.974 & $45 / 5$ & & $46 / 5$ & \\
\hline Total daily energy intake (kcal) & 0.260 & $1,696.0$ & \pm 194.2 & $1,740.0$ & \pm 193.5 \\
\hline Total daily protein $(\mathrm{g})$ & 0.211 & 57.6 & \pm 8.5 & 59.8 & \pm 9.1 \\
\hline \multicolumn{6}{|l|}{ Comorbid conditions } \\
\hline Hypertension & 0.029 & 16 & $32 \%$ & 7 & $14 \%$ \\
\hline Dyslipidemia & 0.295 & 12 & $24 \%$ & 8 & $16 \%$ \\
\hline Ischemic heart disease & 0.653 & 9 & $18 \%$ & 11 & $22 \%$ \\
\hline Congestive heart failure & 0.563 & 5 & $10 \%$ & 7 & $14 \%$ \\
\hline Chronic obstructive pulmonary disease & 0.082 & 0 & 0 & 3 & $6 \%$ \\
\hline Diabetes mellitus & & 32 & $64 \%$ & 32 & $64 \%$ \\
\hline Insulin treatment prior to admission & 0.563 & 5 & $10 \%$ & 7 & $14 \%$ \\
\hline Oral hypoglycemic drugs & 0.613 & 28 & $56 \%$ & 26 & $52 \%$ \\
\hline Metformin treatment & 0.491 & 23 & $46 \%$ & 20 & $40 \%$ \\
\hline \multicolumn{6}{|l|}{ Laboratory findings on admission } \\
\hline Admission blood-glucose concentration $(\mathrm{mg} / \mathrm{dL})$ & 0.108 & 210 & \pm 40 & 224 & \pm 47 \\
\hline Hemoglobin $A_{I c}(\%)$ & 0.269 & 7.9 & \pm 7.1 & 6.8 & \pm 1.2 \\
\hline Total cholesterol (mg/dL) & 0.707 & 202.9 & \pm 14.5 & 204.1 & \pm 17.7 \\
\hline Low-density lipoproteins (mg/dL) & 0.477 & 151.3 & \pm 13.5 & 153.5 & \pm 16.8 \\
\hline High-density lipoproteins (mg/dL) & 0.403 & 49.1 & \pm 6.8 & 50.3 & \pm 7.7 \\
\hline Serum triglycerides (mg/dL) & 0.190 & 194.4 & \pm 27.1 & 202.3 & \pm 32.5 \\
\hline Serum Albumin (gram/dL) & 0.431 & 3.5 & \pm 0.4 & 3.5 & \pm 0.4 \\
\hline
\end{tabular}

Mean admission BG concentration was $224 \pm 47 \mathrm{mg} / \mathrm{dL}$ in the glargine group, not significantly different from the 210 $\pm 40 \mathrm{mg} / \mathrm{dL}$ of the control group $(P=0.108)$. However, during 7 days of ICU admission, mean BG concentrations were significantly lower in the glargine group than the control group (Figure 2A). In contrast, variability in BG levels over the 7 days was similar in both the glargine and control groups (Figure 2B). There were 1,551 episodes of hyperglycemia $>180 \mathrm{mg} / \mathrm{dL}$ in the glargine group (median 25, IQR 7-50) within the 7 days, while there were
2,154 events in the control group (median 35, IQR 13-68; $P<0.001)$. Accordingly, regular insulin usage was significantly less in the glargine group (7.9 U, IQR 6.8-9.8 U) within 7 days, while the controls received a total of 24 (IQR 21-28 units) within the duration of study $(P=0.001)$. Daily use of regular insulin was significantly lower in the glargine group (Figure 3). On the other hand, there were two hypoglycemic patients in the control group, and 15 patients in the glargine group met the hypoglycemic criteria uring the 7 days $(P=0.001$ Table 2$)$. 

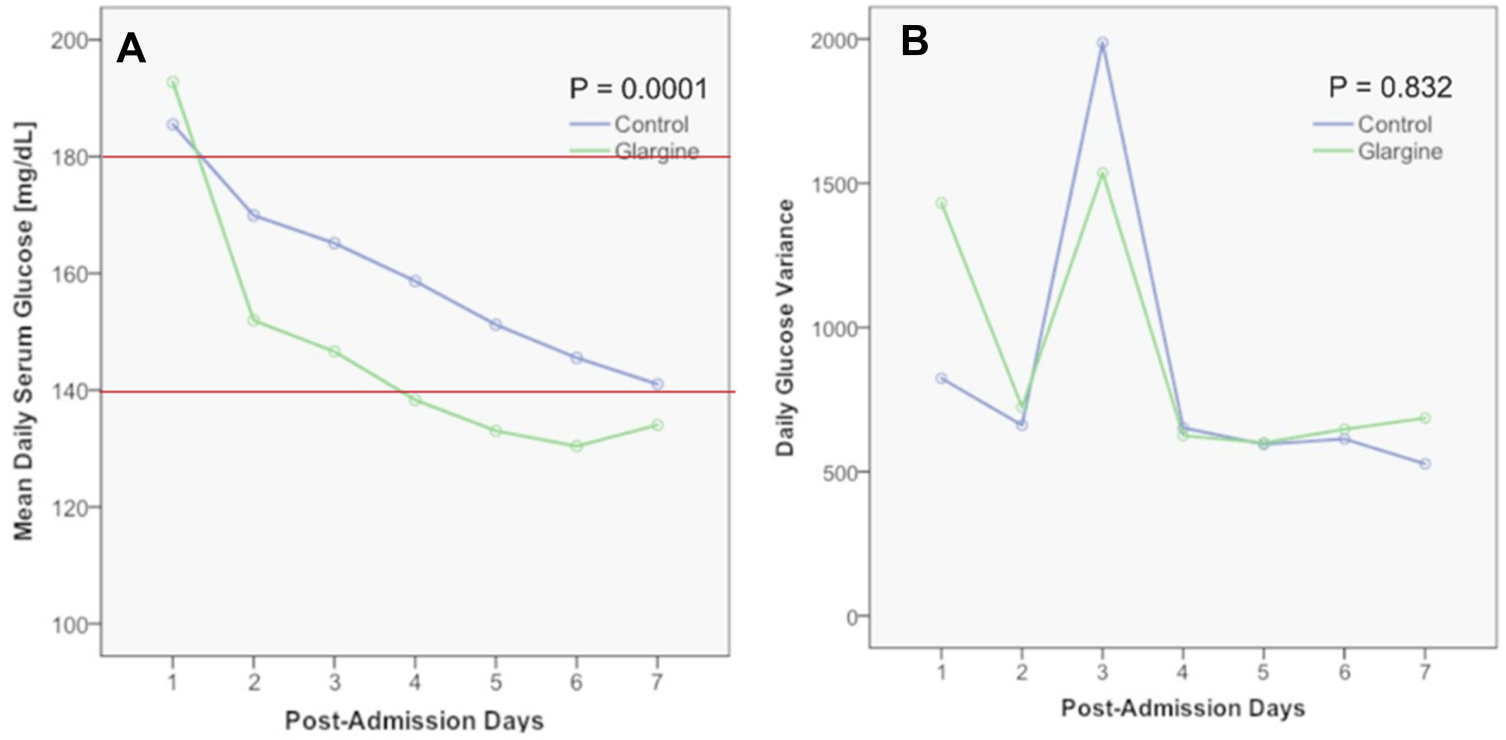

Figure 2 Duration that patients in each group spent in target $(140<$ blood glucose $<180)$ every day for 7 days after admission. (A) Mean daily concentrations of blood glucose; (B) daily variation in measured blood-glucose concentration ( 24-30 hourly measurements). $\mathrm{HbA}_{\mathrm{Ic}}$ was adjusted in the analyses.

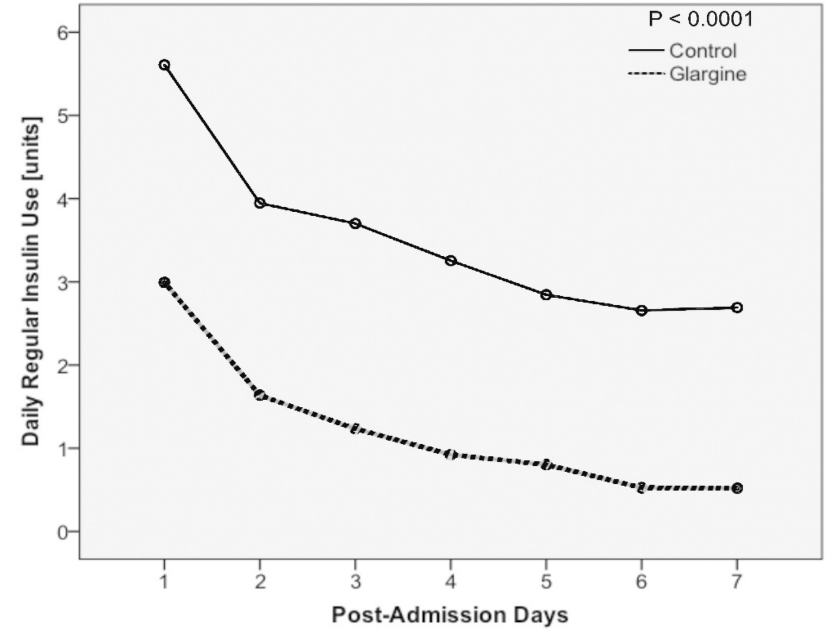

Covariates appearing in the model are evaluated at the following values: $\mathrm{HbA} 1 \mathrm{c}=6.846$

Figure 3 Repeated-measures analysis of daily additional requirement of regular insulin in the two arms of the study.

While patients in the glargine group had significantly shorter ICU stays than the control group $(P=0.018)$ and the duration of mechanical ventilation also shorter in the glargine group $(P=0.023)$, the observed hospital mortality rate was similar in both groups $(P=0.417)$. There were ten patients who developed more than moderate levels of hypoglycemia $(\mathrm{BG}<60 \mathrm{mg} / \mathrm{dL})$ and four with severe hypoglycemia $(\mathrm{BG}<40 \mathrm{mg} / \mathrm{dL})$ over both groups. There was a strong relationship between progressive sepsis and more than moderate levels of hypoglycemia. Clinical outcomes of the patients are shown in Table 3.

\section{Discussion}

This is the first study to use glargine insulin in the management of dysglycemia in critically ill patients during an acute course. Our results showed that insulin glargine may significantly reduce ICU length of stay and duration of mechanical ventilation compared to a control group. However, it did not significantly affect mortality or frequency of hypoglycemic patients in critically ill patients.

Recent guidelines for management of dysglycemia in critically ill patients recommend a target BG of $140-180 \mathrm{mg} / \mathrm{dL}$ with the use of intravenous short-acting insulin. The use of long-acting insulin is not currently recommended for acute management of critically ill patients in ICU settings. $^{12}$ This class of insulin is recommended only for treating hyperglycemia in stable patients in the recovery phase of diabetic ketoacidosis. ${ }^{14,15}$ With non-critically ill who had undergone cardiovascular surgery, Yeldandi et al showed that once-daily insulin glargine was an appropriate method for management of hyperglycemia. ${ }^{16}$ Bhurayanontachai et al showed the same results in stable patients in medical ICUs. ${ }^{17}$ Datta et al showed that oncedaily insulin glargine provided better glycemic control than sliding-scale regular insulin after bariatric surgery. ${ }^{13}$

Contrarily, Ergin et al showed that adding glargine insulin to regular rapid-acting insulin did not have a significant effect on dysglycemia management or its complications, ${ }^{18}$ which may have been because of the lower incidence of hyperglycemia and lower severity scores of the patients included. All 
Table 2 Clinical Outcomes of Patients in Treatment and Control Groups

\begin{tabular}{|l|l|l|l|l|l|}
\hline & P-value & \multicolumn{2}{l|}{ Control (n=55) } & \multicolumn{2}{l|}{ Glargine (n=55) } \\
\hline Death within hospital & 0.455 & 11 & $22 \%$ & 8 & $16 \%$ \\
ICU length of stay (days) & 0.025 & 13.8 & \pm 5.9 & 11.3 & \pm 5.2 \\
Severe composite adverse events & 0.322 & 27 & $54 \%$ & 22 & $44 \%$ \\
Hospital-associated pneumonia & 0.112 & 16 & $32 \%$ & 9 & $18 \%$ \\
Duration of mechanical ventilation (days) & 0.023 & 9.9 & \pm 5.8 & 7.4 & \pm 5.1 \\
Acute kidney injury (AKIN $\geq$ stage I) & 0.654 & 14 & $28 \%$ & 12 & $24 \%$ \\
Vasopressor requirement & $>0.999$ & 9 & $18 \%$ & 9 & $18 \%$ \\
Progression of sepsis & 0.148 & 4 & $8 \%$ & 10 & $20 \%$ \\
Patients with hypoglycemic events & 0.001 & 2 & $4 \%$ & & $29 \%$ \\
Duration of hyperglycemia (hours) & 0.021 & 43.1 & \pm 31.5 & & 30.4 \\
\hline
\end{tabular}

Notes: Duration of hyperglycemia defined by period blood glucose $>180 \mathrm{mg} / \mathrm{dL}$. Frequency of hypoglycemic events defined by blood glucose $<60 \mathrm{mg} / \mathrm{dL}$ presented in this table for total study duration of 7 days and number of patients who had at least one hypoglycemic event. $P$-values for these comparisons obtained through nonparametric Mann-Whitney $U$ tests.

Table 3 Clinical Outcome of Patients According to Development of Hypoglycemia (BG <60 mg/dL) and Severe Hypoglycemia (BG $<40 \mathrm{mg} / \mathrm{dL})$

\begin{tabular}{|c|c|c|c|c|c|}
\hline & $P$-value & \multicolumn{2}{|c|}{$\begin{array}{l}\text { No Hypoglycemia } \\
(n=84)\end{array}$} & \multicolumn{2}{|c|}{$\begin{array}{l}\text { Hypoglycemia } \\
<60 \mathrm{mg} / \mathrm{dL} \\
(\mathrm{n}=17)\end{array}$} \\
\hline Death within hospital & 0.455 & 15 & $18 \%$ & 4 & $24 \%$ \\
\hline ICU length of stay (days) & 0.611 & 12.7 & \pm 5.6 & 11.9 & \pm 6.2 \\
\hline Severe composite adverse events & 0.792 & 40 & $48 \%$ & 9 & $53 \%$ \\
\hline Hospital-associated pneumonia & 0.552 & 22 & $26 \%$ & 3 & $18 \%$ \\
\hline Duration of mechanical ventilation (days) & 0.589 & 8.8 & \pm 5.6 & 8 & \pm 5.7 \\
\hline Acute kidney injury & 0.132 & 19 & $23 \%$ & 7 & $41 \%$ \\
\hline Vasopressor requirement & $>0.999$ & 15 & $18 \%$ & 3 & $18 \%$ \\
\hline \multirow[t]{2}{*}{ Progression of sepsis } & 0.057 & 9 & $11 \%$ & 5 & $29 \%$ \\
\hline & $P$-value & \multicolumn{2}{|c|}{$\begin{array}{l}\text { No Hypoglycemia } \\
(n=94)\end{array}$} & \multicolumn{2}{|c|}{$\begin{array}{l}\text { Hypoglycemia } \\
<40 \mathrm{mg} / \mathrm{dL} \\
(\mathrm{n}=7)\end{array}$} \\
\hline Death within hospital & $>0.999$ & 18 & $19 \%$ & 1 & $14 \%$ \\
\hline ICU length of stay (days) & 0.233 & 12.3 & \pm 5.5 & 15 & \pm 7.6 \\
\hline Severe composite adverse events & 0.636 & 45 & $48 \%$ & 4 & $57 \%$ \\
\hline Hospital-associated pneumonia & 0.678 & 24 & $26 \%$ & 1 & $14 \%$ \\
\hline Duration of mechanical ventilation (days) & 0.319 & 8.5 & \pm 5.5 & 10.7 & \pm 7.1 \\
\hline Acute kidney injury & 0.369 & 23 & $25 \%$ & 3 & $43 \%$ \\
\hline Vasopressor requirement & 0.605 & 16 & $17 \%$ & 2 & $29 \%$ \\
\hline Progression of sepsis & 0.007 & 10 & $11 \%$ & 4 & $57 \%$ \\
\hline
\end{tabular}

Note: $P$-values for these comparisons obtained through nonparametric Mann-Whitney $U$ tests.

these studies were performed in stable critically ill patients and showed safety and efficacy with this intervention. The results of another RCT showed that compared with regular insulin added to parenteral nutrition, glargine insulin had similar glycemic control and rates of hyperglycemia and hypoglycemia in stable critical care patients. ${ }^{19}$ Hakeam et al showed that insulin glargine and regular insulin were both effective methods for BG management in non-critically ill surgical patients with diabetes receiving parenteral nutrition. ${ }^{20}$ They showed that the rate of hypoglycemia was acceptable for both regimens, in contrast to our findings in this study. A recently performed randomized trial in an emergency department evaluated the effect of adding insulin glargine in acute management of diabetic ketoacidosis, and showed that insulin glargine was safe, but further study is needed to define its efficacy in critically ill patients. ${ }^{9}$ The 
authors of this study used insulin glargine in acute management of hyperglycemia in an emergency department, similar to our protocol.

We achieved better results with insulin glargine, which could have been due to the larger sample, different targets, and management of dysglycemia in two studies. Masse et al showed that utilization of long-acting insulin in nondiabetic surgical ICU patients resulted in higher incidence of hypoglycemia, which is in agreement with our results. ${ }^{21}$ Our study showed that there was an insignificant increase in the occurrence of sepsis and acute kidney injury in moderate hypoglycemia and a significant increase in sepsis occurrence in severe hypoglycemia. The higher number of hypoglycemic events in our study may have been due to comorbidities and instability in patient hemodynamics. This can explain why the hypoglycemic patients had severe unstable hemodynamics and received high-dose vasopressor or a combination of vasopressors for hemodynamic stabilization. Not only did this increase in hypoglycemia not have a significant negative clinical effect, nor did it have a significant positive clinical effect regarding patient morbidity. A recently published meta-analysis evaluated the effect of different insulin regimens on glycemic control in hospitalized patients, and showed that in patients receiving enteral feeding mean BG was lower with NPH insulin than glargine + lispro regimens. They recommended additional RCTs with a focus on hard outcomes and severe hypoglycemia, beyond hyperglycemia per se, and thus it is not yet possible to establish the best insulin regimen for management of dysglycemia in critically ill patients. ${ }^{7}$

Controlling BG levels in critically ill patients can be challenging, as there are so many factors that affect glycemic control. Interruptions in nutrition, tapering of steroids or vasopressors, renal replacement therapies, sepsis, and fluctuating stress levels can lead to high variability in BG levels in these patients. If physicians choose to use long-acting insulin, they must first assess the risk factors for dysglycemia in each patient and anticipate changes to concurrent pharmacological therapies and stress levels to anticipate the effect of these factors, as a single episode of hypoglycemia, hyperglycemia, or glucose variability can be an independent risk factor for morbidity and mortality in this population. ${ }^{22}$

\section{Limitations}

Our study was a pilot trial with a small sample in a heterogeneous group of critically ill patients. Due to the fact that it was a pilot trial to obtain preliminary estimates of efficacy and safety, it was felt that performing the entire study in a single-blind fashion with patients being blinded to the randomization arm was adequate, but we need more RCTs with larger samples to generalize the results of this study. We used the Accu-Check device, which is not a calibrated glucometer for hospital care, so this could decrease the accuracy of our glucose measurement. Also, we did not evaluate baseline severity scores like APACHE and SOFA, which could hamper generalization of our results.

\section{Conclusion}

Current evidence shows that an optimal glucose target does not exist for all critically ill patients and that a target should be determined on a case-by-case basis, but hyperglycemia, hypoglycemia, and glucose variability are independent risk factors for mortality in critically ill patients. Based on our findings, insulin glargine reduced the duration of hyperglycemic periods and modestly improved the clinical outcome of critically ill patients during the acute phase of their illness. However,clinicians should be cautioned against increased risks of hypoglycemia events and related adverse outcomes when a longeracting insulin is used for glycemic control in ICU settings.

\section{Data-Sharing Statement}

The data that support the findings of this study are available from the corresponding author upon reasonable request.

\section{Funding}

No funding was requested.

\section{Disclosure}

The authors report no conflicts of interest in this work.

\section{References}

1. van den Berghe G, Wouters P, Weekers F, et al. Intensive insulin therapy in critically ill patients. $N$ Engl J Med. 2001;345:1359-1367. doi:10.1056/NEJMoa011300

2. Lanspa MJ, Dickerson J, Morris AH, Orme JF, Holmen J, Hirshberg EL. Coefficient of glucose variation is independently associated with mortality in critically ill patients receiving intravenous insulin. Crit Care. 2014;18:R86. doi:10.1186/cc13851

3. Mahmoodpoor A, Hamishehkar H, Beigmohammadi M, et al. Predisposing factors for hypoglycemia and its relation with mortality in critically ill patients undergoing insulin therapy in an intensive care unit. Anesth Pain Med. 2016;6:e33849. doi:10.5812/aapm.

4. Mahmoodpoor A, Hamishehkar H, Shadvar K, Beigmohammadi M, Iranpour A, Sanaie S. Relationship between glycated hemoglobin, intensive care unit admission blood sugar and glucose control with ICU mortality in critically ill patients. Indian J Crit Care Med. 2016;20:67-71. doi:10.4103/0972-5229.175938 
5. Samarkandy SJ, Al-Dorzi HM, Tamim HM, Arabi YM. Nursing workload and perception about intensive insulin therapy in critically ill adult patients. Saudi Med J. 2010;31:331-332.

6. Jacobi J, Bircher N, Krinsley J, et al. Guidelines for the use of an insulin infusion for the management of hyperglycemia in critically ill patients. Crit Care Med. 2012;40:3251-3276. doi:10.1097/CCM.0b $013 \mathrm{e} 3182653269$

7. Vercoza Viana M, Vercoza Viana L, Tavares AL, de Azevedo MJ. Insulin regimens to treat hyperglycemia in hospitalized patients on nutritional support: systematic review and meta-analyses. Ann Nutr Metab. 2017;71:183-194. doi:10.1159/000481355

8. Ciardullo AV, Bacchelli M, Daghio MM, Carapezzi C. Effectiveness and safety of insulin glargine in the therapy of complicated or secondary diabetes: clinical audit. Acta Diabetol. 2006;43:57-60. doi:10.1007/s00592-006-0213-7

9. Doshi P, Potter AJ, De Los Santos D, Banuelos R, Darger BF, Chathampally $\mathrm{Y}$. Prospective randomized trial of insulin glargine in acute management of diabetic ketoacidosis in the emergency department: a pilot study. Acad Emerg Med. 2015;22:657-662. doi:10.1111/ acem. 12673

10. Nyenwe EA, Kitabchi AE. Evidence-based management of hyperglycemic emergencies in diabetes mellitus. Diabetes Res Clin Pract. 2011;94:340-351. doi:10.1016/j.diabres.2011.09.012

11. Shankar V, Haque A, Churchwell KB, Russell W. Insulin glargine supplementation during early management phase of diabetic ketoacidosis in children. Intensive Care Med. 2007;33:1173-1178. doi:10. 1007/s00134-007-0674-3

12. Willis GJ, Fisk L, Razak N, Le Compte A, Shaw GM, Chase JG. Insulin glargine in the intensive care unit: A model-based clinical trial design. Biomed Signal Process Control. 2013;8:120-129.

13. Datta S, Qaadir A, Villanueva G, Baldwin D. Once-daily insulin glargine versus 6-hour sliding scale regular insulin for control of hyperglycemia after a bariatric surgical procedure: a randomized clinical trial. Endocr Pract. 2007;13:225-231. doi:10.4158/EP.13. 3.225
14. Andrade-Castellanos CA, Colunga-Lozano LE. [Systematic review with meta-analysis: subcutaneous insulin glargine coadministration for diabetic ketoacidosis]. Gac Med Mex. 2016;152:761-769. Spanish.

15. Houshyar J, Bahrami A, Aliasgarzadeh A. Effectiveness of insulin glargine on recovery of patients with diabetic ketoacidosis: a randomized controlled trial. J Clin Diagn Res. 2015;9:OC01-5. doi:10.7860/JCDR/2015/12005.5883

16. Yeldandi RR, Lurie A, Baldwin D. Comparison of once-daily glargine insulin with twice-daily NPH/regular insulin for control of hyperglycemia in inpatients after cardiovascular surgery. Diabetes Technol Ther. 2006;8:609-616. doi:10.1089/dia.2006.8.609

17. Bhurayanontachai R, Rattanaprapat T, Kongkamol C. Comparison of glycemic control between continuous regular insulin infusion and single-dose subcutaneous insulin glargine injection in medical critically ill patients. Indian J Crit Care Med. 2018;22:174-179. doi:10.4103/ijccm.IJCCM_273_17

18. Ergin AB, Nasr G, Yared JP, Bena J, Nasr C. Glycemic control with use of insulin glargine after cardiothoracic surgery: a retrospective study. Endocr Pract. 2013;19:485-493. doi:10.4158/EP12404.OR

19. Oghazian MB, Javadi MR, Radfar M, et al. Effectiveness of regular versus glargine insulin in stable critical care patients receiving parenteral nutrition: a randomized controlled trial. Pharmacotherapy. 2015;35:148-157. doi:10.1002/phar.2015.35.issue-2

20. Hakeam HA, Mulia HA, Azzam A, Amin T. Glargine insulin use versus continuous regular insulin in diabetic surgical noncritically ill patients receiving parenteral nutrition: randomized controlled study. JPEN J Parenter Enteral Nutr. 2017;41:1110-1118. doi:10.1177/ 0148607116644710

21. Masse J, Giuliano CA, Brown S, Paxton RA. Association between the use of long-acting insulin and hypoglycemia in nondiabetic patients in the surgical intensive care unit. J Intensive Care Med. 2018;33:317-321. doi:10.1177/0885066616677030

22. Krinsley JS, Grover A. Severe hypoglycemia in critically ill patients: risk factors and outcomes. Crit Care Med. 2007;35:2262-2267. doi:10.1097/01.CCM.0000282073.98414.4B

\section{Publish your work in this journal}

Diabetes, Metabolic Syndrome and Obesity: Targets and Therapy is an international, peer-reviewed open-access journal committed to the rapid publication of the latest laboratory and clinical findings in the fields of diabetes, metabolic syndrome and obesity research. Original research, review, case reports, hypothesis formation, expert opinion and commentaries are all considered for publication. The manuscript management system is completely online and includes a very quick and fair peer-review system, which is all easy to use. Visit http://www.dovepress.com/testimonials.php to read real quotes from published authors.

Submit your manuscript here: https://www.dovepress.com/diabetes-metabolic-syndrome-and-obesity-targets-and-therapy-journal 